\title{
A multi-depot traveling salesman problem and its iterative and integrated approaches
}

\section{William $\mathrm{Ho}^{*}$}

Operations and Information Management Group

Aston Business School, Aston University

Birmingham B4 7ET, United Kingdom

E-mail:w.ho@aston.ac.uk

* Corresponding author

\section{Ping Ji}

Department of Industrial and Systems Engineering,

The Hong Kong Polytechnic University,

Hung Hom, Kowloon, Hong Kong

E-mail:mfpji@polyu.edu.hk

Prasanta K. Dey

Operations and Information Management Group

Aston Business School, Aston University

Birmingham B4 7ET, United Kingdom

E-mail: p.k.dey@aston.ac.uk

\begin{abstract}
This paper formulates a logistics distribution problem as the multi-depot traveling salesman problem (MDTSP). The decision makers not only have to determine the traveling sequence of the salesman for delivering finished products from a warehouse or depot to a customer, but also need to determine which depot stores which type of products so that the total traveling distance is minimized. The MDTSP is similar to the combination of the traveling salesman and quadratic assignment problems, which are the two of the most commonly studied optimization problems in operations research due to their wide applications. Each individual problem is already complex and difficult to solve due to its NP-hard nature. In this paper, the two individual hard problems or models are formulated first. Then, the problems are integrated together, that is, the MDTSP of which the complexity is absolutely much higher than that of the individual ones. The MDTSP is constructed as both integer nonlinear and linear programming models. After formulating the models, we verify the integrated models using commercial packages, and most importantly, investigate whether an iterative approach, that is, solving the individual models repeatedly, can generate an optimal solution to the MDTSP.
\end{abstract}


Keywords: Logistics distribution management; Mathematical modeling; Optimization; Quadratic assignment problem; Traveling salesman problem.

Biographical notes: William Ho is a Lecturer in the Aston Business School, The Aston University. He obtained $\mathrm{BE}$ and $\mathrm{PhD}$ degrees from the Department of Industrial and Systems Engineering, The Hong Kong Polytechnic University. His research areas include the applications of operations management and operations research techniques in the printed circuit board assembly, supply chain management/logistics, and higher education management processes.

Ping Ji is an Associate Professor in the Department of Industrial and Systems Engineering, The Hong Kong Polytechnic University. He has over 50 papers in various international journals, such as, IIE Transactions, Transactions of the ASME: Journal of Manufacturing Science and Engineering, IEEE Transactions on Robotics and Automation, European Journal of Operational Research, Journal of Operational Research Society. His research areas include production management, operations research, and CAD/CAM.

Prasanta Kumar Dey is a Senior Lecturer in the Aston Business School, The Aston University. He has a bachelor of engineering and $\mathrm{PhD}$ (engineering) from Jadavpur University, India and a Master's in industrial engineering from Asian Institute of Technology, Thailand. Prior to joining Aston Business School in 2004, he was with the University of the West Indies in Barbados and Indian Oil Corporation Limited in India. He has published extensively in refereed journals like International Journal of Operations and Production Management, International Journal of Production Economics, International Journal of Project Management, IEEE Transactions on Engineering Management, Applied Mathematical Modelling etc. His current research includes application of multiple criteria decision making in both manufacturing and service industry. 


\section{Introduction}

\subsection{Background}

The distribution of finished products from warehouses or depots to customers is a practical and challenging problem in logistics management. Better scheduling decision can result in higher level of productivity, and higher level of customer satisfaction because more customers are served in a shorter time. The distribution problem studied in this paper is formulated as the traveling salesman problem (TSP) with multiple depots or called as the multi-depot TSP (MDTSP). In the well-known TSP for the distribution problem, there is no limitation on the capacity of the vehicle, and the products ordered by the customers are stored in the same depot. The salesman can, therefore, deliver the products from a depot to all customers in a single route without returning to the depot (DePuy et al., 2005). In the real-world situations, however, a logistics company may have more than one depot for storing the products. In addition, different customers may order different types of products, which are stored in different depots. In such cases, the MDTSP instead of the TSP should be applied to. The MDTSP is described as follows. Consider a company has multiple depots with known location and unlimited capacity, a vehicle with known capacity, and a set of customers with known demand and location. Each customer orders a unique type of products with a large volume so that a vehicle can only serve one customer in each route. The number of depots is equivalent to the number of product types ordered by the customers. Each product type is stored in a depot exactly. In the MDTSP, the vehicle starts and finishes at its original location. The decision makers of the company not only need to determine which customer is served first, second, and so on, that is, the customer scheduling problem but also which product types are stored in which depots, that is, the depot assignment problem. The objective of the MDTSP is to minimize the total distance traveled or time spent by the vehicle for serving all customers.

Since the customer scheduling and depot assignment problems are similar to the TSP and the quadratic assignment problem (QAP), respectively, the MDTSP can be regarded as an integration of both the TSP and the QAP. Actually, the individual problems are already very complex and hard to solve (Burkard et al., 1991; Reinelt, 1994). Hence, the MDTSP is extremely complicated and very challenging. Although heuristic methods and simulation are alternative tools for solving the problem, no one can assure that the solution generated using these tools is optimal or even no one knows how good the solution is before the optimal solution has been found. So, in this paper, the focus is confined to obtaining the optimal solution to the MDTSP. In order to achieve this goal, two possible approaches can be adopted called the iterative and integrated. In the iterative approach, the MDTSP is decomposed into 
the TSP-like and QAP-like problems first. The individual problems are then solved repeatedly until there is no improvement on the solution's quality. The second approach is an integrated way, which solves the integrated mathematical model of the MDTSP. One of the motivating factors for adopting the iterative approach is its simplicity because only one single problem instead of two is focused on at a time. Nevertheless, a question is: Can this approach generate the optimal solution to the original problem? To answer this critical question, an investigation on the effectiveness of the iterative approach is, therefore, carried out in this paper.

There are extensive variations of the TSP: the asymmetric TSP (Choi et al., 2003), the generalized TSP (Snyder and Daskin, 2005), the multiple TSP (Bektas, 2005), the period TSP (Bertazzi et al., 2004), the pickup and delivery TSP (Renaud et al., 2002), the probabilistic TSP (Bianchi et al., 2005), the TSP with precedence constraints (Moon et al., 2002), the TSP with time window (Jula et al., 2005), and so on. The definitions of all TSP variants are described in Table 1. To our knowledge, however, there is no publication in the international literature studying the MDTSP. The lack of research contribution is the primal motivation for this paper.

\subsection{The notation used in the mathematical models}

Before formulating the individual and integrated models for the MDTSP, the notation used is described in this sub-section. Consider a group of customers $(i, j=1,2, \ldots, m)$ is served by a salesman or vehicle. Each customer orders a unique type of products $(p=1,2, \ldots, n)$. Each of the $n$ product types must be stored in a depot, say $q$. But, a depot can only store a particular type of products. Since a product type must be assigned to a depot, $n$ depots are needed to store $n$ types of products, that is, $q=1,2, \ldots, n$. The quantity of products ordered by each customer is so large that the vehicle can only serve a customer in each route. The problem faced can be stated as follows. At the beginning, the vehicle starts from its original location, moves to a depot that stores products, picks up products of the same type from the depot, then moves to the desired location of the customer, and delivers the products there. After that, the vehicle moves back to the previous depot if the next customer orders the same product type as the previous one or moves to another depot if it is different from the previous one to pick up the next product and repeats the distribution procedure. After delivering all products to the customers, the vehicle returns to its original location. In order to minimize the total distance traveled by the vehicle for serving all customers, it is necessary to determine not only the customer scheduling, that is, $x_{i j}$ but also the depot assignment, that is, $y_{p_{j} q}$ in which $p_{j}$ refers to the product type of customer $j$. All notation used is summarized in Table 2. 
This paper is organized as follows. Sections 2 and 3 discuss the customer scheduling and depot assignment problems, respectively. Section 4 integrates both individual problems together. The MDTSP in the form of nonlinear programming model is formulated first. The nonlinear type model is then converted equivalently to the integer linear programming model. Section 5 compares the iterative and integrated approaches for solving the MDTSP. A commercial package called CPLEX is used to generate the optimal solution of the MDTSP in the linear form, and solve the individual problems. Finally, Section 6 concludes the paper.

\section{The customer scheduling problem}

The problem of determining the delivering sequence of the vehicle or the customer scheduling problem can be formulated as the TSP-like model. The TSP, which is one of the most widely studied integer programming problems, can be easily stated as follows. A salesman (or vehicle) wants to visit $m$ distinct cities (or customers) and then returns home. He wants to determine the sequence of the travel so that the overall traveling distance is minimized while visiting each city not more than once. Although the TSP is conceptually simple, it is difficult to obtain an optimal solution. In an $m$-city situation, any permutation of $m$ cities yields a possible solution. Thus, $m$ ! possible tours must be evaluated in the search space. By introducing variables $x_{i j}$ to represent the tour of the salesman travels from city $i$ to city $j$, one of the integer programming formulations for the TSP can be written as (Winston and Venkataramanan, 2005):

$$
\text { Minimize } z=\sum_{i=0}^{m} \sum_{\substack{j=0 \\ j \neq i}}^{m} d_{i j} x_{i j}
$$

subject to

$$
\begin{array}{lr}
\sum_{i=0}^{m} x_{i j}=1 & j=0,1, \ldots, m ; i \neq j . \\
\sum_{j=0}^{m} x_{i j}=1 & i=0,1, \ldots, m ; i \neq j . \\
u_{i}-u_{j}+m x_{i j} \leq m-1 & i, j=1,2, \ldots, m ; i \neq j . \\
\text { All } x_{i j}=0 \text { or } 1, \text { All } u_{i} \geq 0 \text { and is a set of integers }
\end{array}
$$

The distance between city $i$ and city $j$ is denoted as $d_{i j}$. The objective function (1) is simply to minimize the total distance traveled in a tour. Constraint set (2) ensures that the salesman arrives once at each city. Constraint set (3) ensures that the salesman leaves each city once. Constraint set (4) is to avoid the presence of sub-tour. 
Generally, the TSP formulated is known as the Euclidean TSP, in which the distance matrix $d$ is expected to be symmetric, that is $d_{i j}=d_{j i}$ for all $i, j$, and to satisfy the triangle inequality, that is, $d_{i k} \leq d_{i j}+d_{j k}$ for all distinct $i, j, k$.

In the TSP, the vehicle delivers products from one customer to another directly without returning to the depot until all customers are visited. The objective is, therefore, the minimization of $\sum_{i=0}^{m} \sum_{\substack{j=0 \\ j \neq i}}^{m} d_{i j} x_{i j}$. Herein, the original location of the vehicle is denoted as “ 0 ".

The objective function needs to be modified for the MDTSP because the vehicle must pick up the products from a depot before visiting the next customer. Suppose the assignment of product types to depots, that is, the depot assignment problem is solved beforehand, the customer scheduling model can then be formulated as follows:

$$
\text { Minimize } z=\sum_{i=0}^{m} \sum_{\substack{j=1 \\ j \neq i}}^{m} \sum_{q=1}^{n}\left(d_{i q}+d_{q j}\right) x_{i j}+\sum_{i=1}^{m} d_{i 0} x_{i 0}
$$

subject to

$$
\text { (2), (3), and (4) }
$$

$$
\text { All } x_{i j}=0 \text { or } 1, \text { All } u_{i} \geq 0 \text { and is a set of integers }
$$

Since M2 contains both binary variables, that is, $x_{i j}$ as well as integer variables, that is, $u_{i}$, it can be regarded as a pure integer linear programming model. The objective function (5) is to minimize the total traveling distance of the vehicle for serving all customers:

- $\quad$ The distance between the location of customer $i$ and depot $q$ (if $i=0$, it is the distance between the original location at the beginning and depot $q$ );

- $\quad$ The distance between depot $q$ and the location of the next customer $j$;

- $\quad$ The distance between the location of the last customer $i$ and the original location at the end.

If the moving speed of the vehicle is incorporated, then the objective can be to minimize the total delivering time. Constraint sets (2) and (3) ensure that each customer is served by the vehicle exactly once. Constraint set (4) is to avoid the occurrence of sub-tours. Since the original location of the vehicle must be visited first, it is redundant to include $i$ and/or $j=0$ in constraint set (4). Although the constraint set (4) is able to guarantee the solution generated is feasible, it increases the complexity of the model as there are $m(m-1)$ constraints in this sub-tour elimination constraint. Since M2 is very similar to M1 except the objective function, it is called the TSP-like model. 


\section{The depot assignment problem}

In the MDTSP, it is necessary to determine which depot stores which types of products or the depot assignment problem. Besides, each type of products is assigned to a single depot. The problem can be formulated as the QAP-like model. The QAP is a generalization of the linear assignment problem. The major difference between them is that the objective function of the QAP is in nonlinear expression. It is, therefore, more sophisticated and difficult to solve. The QAP is described as follows. Consider a set of facilities $(p, r=1,2, \ldots, n)$ is placed uniquely in a set of locations $(q, s=1,2, \ldots, n)$. It is noted that the facilities and locations refer to the products types and depots in the MDTSP, respectively. The workflow intensity between each pair of facilities is $a_{p r}$ while the distance between each pair of locations is $b_{q s}$. Also, a fixed cost $c_{p q}$ associated with the placement of facility $p$ in location $q$ is specified. The formulation of the QAP can be written as (Burkard et al., 1991; Williams, 1999):

$$
\text { Minimize } z=\sum_{p=1}^{n} \sum_{q=1}^{n} \sum_{\substack{r=1 \\ r \neq p}}^{n} \sum_{\substack{s=1 \\ s \neq q}}^{n} a_{p r} b_{q s} y_{p q} y_{r s}+\sum_{p=1}^{n} \sum_{q=1}^{n} c_{p q} y_{p q}
$$

subject to

$$
\begin{array}{ll}
\sum_{p=1}^{n} y_{p q}=1 & q=1,2, \ldots, n . \\
\sum_{q=1}^{n} y_{p q}=1 & p=1,2, \ldots, n . \\
\text { All } y_{p q}=0 \text { or } 1 &
\end{array}
$$

The decision variables $y_{p q}$ represent the placement of facility $p$ in location $q$. The objective function (6) is to assign facilities to locations so that the traveling distance of material flow is minimized, while assuming that the cost of assigning a facility does not depend upon the location, that is, $c_{p q}=0$. Constraint set (7) ensures that each location must only be placed by one facility. Constraint set (8) ensures that each facility must be assigned to one location only.

In the depot assignment problem, the goal is to assign the product types to depots in such a way that the total distance traveled by the vehicle is minimized. Thus, the objective function (6) is not suitable for the problem. If the delivering sequence of the vehicle is known, that is, $x_{i j}$ in M2 is known, the mathematical model for the depot assignment problem can be formulated in the following: 
Minimize $z=\sum_{i=0}^{m} \sum_{\substack{j=1 \\ j \neq i}}^{m} \sum_{p=1}^{n} \sum_{q=1}^{n}\left(d_{i q}+d_{q j}\right) y_{p_{j} q}+d_{i 0}$

subject to

(7), and (8)

All $y_{p q}=0$ or 1

Since M4 only contains binary variables, that is, $y_{p q}$, it can be regarded as a binary integer linear programming model. Similar to M2, the objective function (9) is to calculate the total distance traveled by the vehicle for serving all customers. Constraint sets (7) and (8) ensure that a depot is used to store a particular type of products exactly.

\section{The multi-depot traveling salesman problem}

Before solving M2, it is essential to obtain the solution of the depot assignment problem first. On the other hand, M4 cannot be solved until the solution of the customer scheduling problem is known. It is no doubt that the customer scheduling problem and the depot assignment problem are closely related. Moreover, the objective function in M2 and M4 is to minimize the total traveling distance of the vehicle. It can be noticed that the amount of distance traveled is dependent on which customer is visited next together with where the next required product type is stored. In order to obtain the optimal solution, both problems should be, therefore, considered and solved simultaneously. A complete mathematical model for the integration of both customer scheduling and depot assignment problems can be formulated as follows:

$$
\text { Minimize } z=\sum_{i=0}^{m} \sum_{\substack{j=1 \\ j \neq i}}^{m} \sum_{p=1}^{n} \sum_{q=1}^{n}\left(d_{i q}+d_{q j}\right) x_{i j} y_{p_{j} q}+\sum_{i=1}^{m} d_{i 0} x_{i 0}
$$

subject to

$$
\text { (2), (3), (4), (7), and (8) }
$$

All $x_{i j}$ and $y_{p q}=0$ or 1 ; All $u_{i} \geq 0$ and is a set of integers

M5 can be regarded as a pure integer nonlinear programming model because there is a nonlinear term, that is, $x_{i j} y_{p_{j} q}$ in the objective function and the model contains both binary variables, that is, $x_{i j}$ and $y_{p q}$ and integer variables, that is, $u_{i}$. The interpretation of the objective function and constraint sets has been mentioned in Sections 2 and 3. 
The model is very complex and challenging since both customer scheduling and depot assignment problems are considered simultaneously. It will, therefore, require a lot of computational time to solve the model, and to verify the model. It is, however, found that many binary decision variables are defined. So, it can be reformulated as the linear programming model using the following steps:

- $\quad$ Introducing a binary variable $w$ to replace the product term $x y$;

- $\quad$ Using the extra constraints: $w \leq x, w \leq y$, and $w \geq x+y-1$ to reflect the logical condition: $w=1$ if and only if $x=1$ and $y=1$.

For M5, the nonlinear term in the objective function is in the form of products of two binary variables. It can be, therefore, rewritten as a linear one by introducing an extra binary variable $w_{i j q}$ as well as three extra constraint sets. The interpretation of the decision variable $w_{i j q}$ is:

$$
w_{i j q}= \begin{cases}1 & \text { if customer } j \text { is visited just after customer } i \text { and } \\
\text { the product ty pe ordered by customer } j \text { is stored in depot } q \\
0 \quad \begin{array}{l}
\text { otherwise. }
\end{array}\end{cases}
$$

After incorporating the extra decision variable and constraint sets, M5 can be converted into a linear programming model as follows:

$$
\text { Minimize } z=\sum_{i=0}^{m} \sum_{\substack{j=1 \\ j \neq i}}^{m} \sum_{q=1}^{n}\left(d_{i q}+d_{q j}\right) w_{i j q}+\sum_{i=1}^{m} d_{i 0} x_{i 0}
$$

subject to

$$
(2),(3),(4),(7),(8), \text { and }
$$

$$
\begin{array}{ll}
w_{i j q} \leq x_{i j} & \text { for } i=0,1, \ldots, m ; \\
& \text { for } j=1,2, \ldots, m ; i \neq j ; \\
& \text { for } q=1,2, \ldots, n . \\
w_{i j q} \leq y_{p_{j} q} & \text { for } i=0,1, \ldots, m ; \\
& \text { for } j=1,2, \ldots, m ; i \neq j ; \\
& \text { for } p, q=1,2, \ldots, n . \\
w_{i j q} \geq x_{i j}+y_{p_{j} q}-1 & \text { for } i=0,1, \ldots, m ; \\
& \text { for } j=1,2, \ldots, m ; i \neq j ; \\
& \text { for } p, q=1,2, \ldots, n .
\end{array}
$$

All $x_{i j}, y_{p q}$, and $w_{i j q}=0$ or 1 ; All $u_{i} \geq 0$ and is a set of integers 
Since all the polynomial expressions are converted into linear expressions in M6, it can be regarded as a pure integer linear programming model. In M6, the objective function (11) and constraint sets (12) to (14) are the linear expression of the objective function (10) of M5. Although the model becomes linear, both numbers of constraints and variables in M6 are much greater than that in M5. For the number of variables, $m^{2} n$ of $w_{i j q}$ are introduced in M6 besides $\left(m^{2}+m+n^{2}\right)$ binary variables and $m$ integer variables. For the number of constraints, besides $\left(m^{2}+m+2 n+2\right)$ constraints, M6 has $3 m^{2} n$ constraints more for constraint sets (12) to (14).

In order to understand the MDTSP clearly and compare which formulation is better in terms of the computational time spent for achieving the global optimum, a numerical example with four customers and four different product types is applied. The data of the MDTSP is listed in Table 3, whereas the coordinates of the original location are $(0,0)$. Two commercial packages are used in this paper to solve the mathematical models. Firstly, BARON is a computational system for solving non-convex optimization problems including the pure integer nonlinear programming models to global optimality. So, it is adopted to solve M5. Secondly, CPLEX is a well-known powerful integer linear programming solver. It is, therefore, applied to solving M6. By these two commercial packages, both M5 and M6 are tested by solving the MDTSP example shown in Table 3, and both generate the same optimal solution. The optimal delivering sequence of the vehicle is: original location $\rightarrow d_{3}(\operatorname{depot} 3) \rightarrow c_{3}$ (customer 3 ) $\rightarrow f_{4} \rightarrow c_{4} \rightarrow f_{2} \rightarrow c_{2} \rightarrow f_{1} \rightarrow c_{1} \rightarrow$ original location, whereas the total distance traveled by the vehicle is 310.26 units. The "solve summaries" of both M5 and M6 are listed in Appendices I and II, respectively. According to the "solve summaries", it is found that the pure integer linear programming model (M6) is more desirable than the pure integer nonlinear programming model (M5) in terms of the amount of computational time spent, $0.16 \mathrm{~s}$ vs. 1.68s.

\section{$5 \quad$ Iterative approach vs. integrated approach}

Solving the individual customer scheduling and depot assignment problems repeatedly is an alternative approach to optimize the MDTSP. This iterative approach is simpler than the integrated approach because only one single problem instead of two is focused on at a time. Nevertheless, the iterative approach may lead to a local optimal solution, and a question "Can this approach guarantee that the solution generated is globally optimal?" is raised. In order to provide valid evidence to answer this query, the effectiveness of the iterative approach is investigated using the same MDTSP example shown in Table 3. The results of the investigation, definitely, direct us to an appropriate way, that is, either iterative or integrated for optimizing the MDTSP. 
The iterative approach, as illustrated in Figure 1, is adopted in which the customer scheduling model, that is, M2 is solved beforehand while assuming that the assignment of product types to depots is predetermined. After that, the depot assignment model, that is, M4 is solved based on the optimal sequence of customer visits to find out the minimum traveling distance. The individual models are solved sequentially by CPLEX because they are in the linear form. If there is an improvement on the solution's quality, the above procedure will be repeated again. The final best solution obtained by the approach is compared with the global optimal solutions obtained by the integrated mathematical models, that are, M5 and M6.

Suppose the assignment of product types to depots is generated arbitrarily that product types $1,3,2$, and 4 are stored in depots $1,2,3$, and 4 , respectively, that is, $y_{11}=y_{32}=y_{23}=y_{44}=$ 1. In this case, the procedure is repeated two times more, that is, totally three iterations. The solutions together with the objective value in each of the three iterations are summarized in Table 4. The objective value remains the same as 314.11 units after the third iteration, the procedure is, therefore, terminated. Although the individual models are solved sequentially several times, this iterative approach cannot generate the global optimal solution to the integrated problem, that is, 310.26 units.

Besides the MDTSP example shown in Table 3, three sets of MDTSP are generated randomly in order to examine the effectiveness of the iterative approach. The comparison between the iterative and integrated approaches for the MDTSPs is summarized in Table 5. According to the results, it is proved that the iterative approach is unable to guarantee that the solutions are globally optimal. In addition, the larger the problem size, the poorer the quality of the solutions generated by the iterative approach.

Based on the above observations, it is concluded that the customer scheduling and depot assignment problems must be integrated together in order to optimize the MDTSP or find out the shortest distance traveled by the vehicle. Besides, it is noticed that the initial depot assignment plays a vital role in the approach. If the initial depot assignment is not made carefully, even if the customer scheduling problem is solved to optimality, it could result in a poor performance. 


\section{Conclusions}

The TSP and its variants have been studied extensively because of their wide applications. Nevertheless, there is a rigid assumption that there is only one depot. If a logistics company has more than one depot, they are not appropriate. To resolve this limitation, this paper focused on the TSP with multiple depots or so-called multi-depot TSP (MDTSP). The MDTSP is more practical, while at the same time more challenging because the decision makers not only have to determine which customers are visited first, second, and so on (the customer scheduling problem) but also the assignment of product types to depots (the depot assignment problem) so that the total traveling distance is the shortest. This paper formulated the customer scheduling and depot assignment problems as the TSP-like and QAP-like models, respectively. It was noticed that the problems are inter-related, and inseparable. One cannot be solved unless the solution of the other one is obtained beforehand. Due to their inter-related relationship, two integrated mathematical models in the nonlinear and linear forms were constructed for the MDTSP. Furthermore, we proved that the iterative approach, that is, sequentially solving the individual customer scheduling and depot assignment models is not the best way to optimize the MDTSP because the approach cannot guarantee that the solution is globally optimal. The customer scheduling and depot assignment problems must be, therefore, integrated together in order to minimize the total distance traveled by the vehicle.

\section{Acknowledgment}

The work described in this paper was partially supported by a grant from the Research Grants Council of Hong Kong Special Administrative Region, China (Project No. PolyU 5259/04E). Besides, the authors would like to thank the reviewer for the valuable comments on this paper. 


\section{References}

Bektas, T. (2005) 'The multiple traveling salesman problem: an overview of formulations and solution procedures', Omega-The International Journal of Management Science. (forthcoming).

Bertazzi, L., Paletta, G. and Speranza, M.G. (2004) 'An improved heuristic for the period traveling salesman problem', Computers \& Operations Research, Vol. 31, pp.1215-1222.

Bianchi, L., Knowles, J.K. and Bowler, N. (2005) 'Local search for the probabilistic traveling salesman problem: correction to the 2-p-opt and 1-shift algorithms', European Journal of Operational Research, Vol. 162, pp.206-219.

Burkard, R.E., Karisch, S.E. and Rendl, F. (1991) 'QAPLIB - A quadratic assignment problem library', European Journal of Operational Research, Vol. 55, pp.115-119.

Choi, I.C., Kim, S.I. and Kim, H.S. (2003) 'A genetic algorithm with a mixed region search for the asymmetric traveling salesman problem', Computers \& Operations Research, Vol. 30, pp.773-786.

DePuy, G.W., Moraga, R.J. and Whitehouse, G.E. (2005) 'Meta-RaPS: a simple and effective approach for solving the traveling salesman problem', Transportation Research Part E, Vol. 41, pp.115-130.

Jula, H., Dessouky, M., Ioannou, P. and Chassiakos, A. (2005) 'Container movement by trucks in metropolitan networks: modeling and optimization', Transportation Research Part E, Vol. 41, pp.235-259.

Moon, C., Kim, J., Choi, G. and Seo, Y. (2002) 'An efficient genetic algorithm for the traveling salesman problem with precedence constraints', European Journal of Operational Research, Vol. 140, pp.606-617.

Reinelt, G. (1994) The Traveling Salesman: Computational Solutions for TSP Applications, Springer, New York.

Renaud, J., Boctor, F.F. and Laporte, G. (2002) 'Perturbation heuristics for the pickup and delivery traveling salesman problem', Computers \& Operations Research, Vol. 29, pp.1129-1141.

Snyder, L.V. and Daskin, M.S. (2005) 'A random-key genetic algorithm for the generalized traveling salesman problem', European Journal of Operational Research. (forthcoming).

Williams, H.P. (1999) Model Building in Mathematical Programming, Wiley, New York.

Winston, W.L. and Venkataramanan, M. (2003) Introduction to Mathematical Programming: Operations Research, Brooks/Cole-Thomson Learning, California. 
Figure 1 The iterative approach

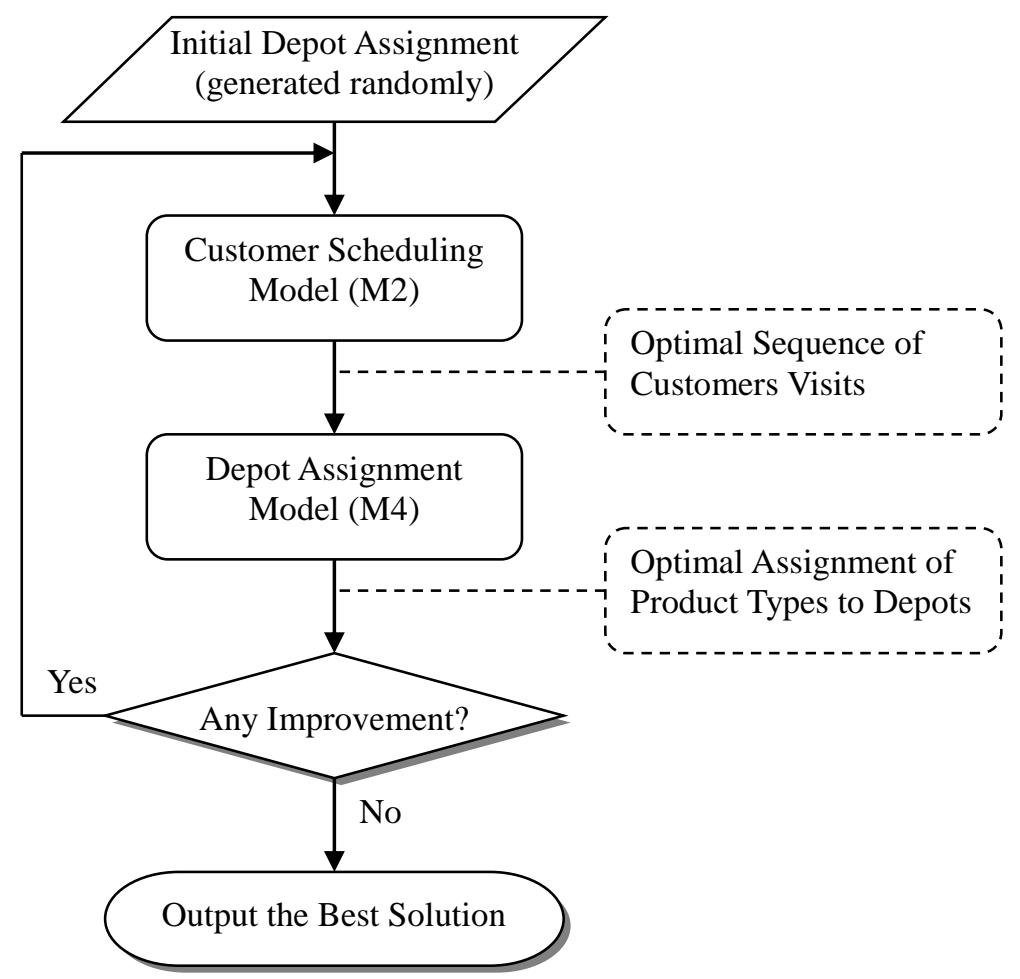


Table 1 Definitions of the TSP variants

\begin{tabular}{|c|c|}
\hline TSP Variants & Definitions \\
\hline Asymmetric TSP & $\begin{array}{l}\text { The bi-directional distances between a pair of } \\
\text { customers are not necessarily identical }\end{array}$ \\
\hline Generalized TSP & $\begin{array}{l}\text { The customers are divided into several groups, and not } \\
\text { all customers need to be visited }\end{array}$ \\
\hline Multiple TSP & $\begin{array}{l}\text { More than one salesman is allowed to be used, and } \\
\text { each customer is served by a salesman only }\end{array}$ \\
\hline Period TSP & $\begin{array}{l}\text { The vehicle has to visit each customer several times } \\
\text { over a given period of time }\end{array}$ \\
\hline Pickup and Delivery TSP & $\begin{array}{l}\text { The vehicle may both receive products from the } \\
\text { customers and deliver products to the customers at the } \\
\text { same time }\end{array}$ \\
\hline Probabilistic TSP & $\begin{array}{l}\text { Each customer has a predetermined probability of } \\
\text { requiring a visit }\end{array}$ \\
\hline TSP with Precedence Constraints & There exist a delivering order between the customers \\
\hline TSP with Time Window & $\begin{array}{l}\text { The vehicles must arrive at the customers before the } \\
\text { latest arrival time, while arriving before the earliest } \\
\text { arrival time results in waiting }\end{array}$ \\
\hline
\end{tabular}


Table 2 Notation

\section{Indices:}

$i, j$ : customers $(i, j=0,1, \ldots, m)$.

$p$ : product types $(p=1,2, \ldots, n)$.

$q$ : depots $(q=1,2, \ldots, n)$.

\section{Distances:}

$d_{0 q}$ : distance traveled from vehicle's original location to depot $q$.

$d_{q j}$ : distance traveled from depot $q$ to the location of customer $j$.

$d_{i q}$ : distance traveled from the location of customer $i$ to depot $q$.

$d_{i 0}$ : distance traveled from the location of customer $i$ to original location.

\section{Sub-tour Elimination Constraint:}

$u_{i}$ : delivering order of customer $i$.

\section{Decision Variables:}

$x_{i j}=1$ if customer $i$ is visited immediately prior to customer $j ; 0$ otherwise.

$y_{p_{j} q}=1$ if product type $p$ ordered by customer $j$ is stored in depot $q ; 0$ otherwise. 
Table 3 Data of the MDTSP

\begin{tabular}{ccccccc}
\hline $\begin{array}{c}\text { Customers } \\
(i)\end{array}$ & Types & \multicolumn{2}{c}{ Coordinates } & Depots & \multicolumn{2}{c}{ Coordinates } \\
& $\left(p_{i}\right)$ & $x$ & $y$ & $(q)$ & $x$ & $y$ \\
\hline 1 & 4 & 30 & 40 & 1 & 10 & 30 \\
2 & 3 & 30 & 60 & 2 & 10 & 20 \\
3 & 1 & 50 & 20 & 3 & 20 & 10 \\
4 & 2 & 50 & 40 & 4 & 30 & 10 \\
\hline
\end{tabular}


Table 4 Best solutions obtained in each iteration of the iterative approach

\begin{tabular}{llll}
\hline & Models & Solutions & Objective values \\
\hline 1st iteration: & M2 & $x_{10}=x_{31}=x_{42}=x_{23}=x_{04}=1$ & 333.88 \\
& M4 & $y_{31}=y_{12}=y_{23}=y_{44}=1$ & 329.16 \\
2nd iteration: & M2 & $x_{20}=x_{31}=x_{12}=x_{43}=x_{04}=1$ & 327.37 \\
& M4 & $y_{31}=y_{22}=y_{43}=y_{14}=1$ & 314.24 \\
3rd iteration: & M2 & $x_{30}=x_{01}=x_{12}=x_{43}=x_{24}=1$ & 314.11 \\
& M4 & $y_{31}=y_{22}=y_{43}=y_{14}=1$ & 314.11 \\
\hline
\end{tabular}


Table 5 Comparison of the iterative and integrated approaches for different problem sizes

\begin{tabular}{cccc}
\hline Problems $(\boldsymbol{m} \times \boldsymbol{n})$ & $\begin{array}{c}\text { Best Solutions by } \\
\text { Iterative Approach }\end{array}$ & $\begin{array}{c}\text { Optimal Solutions by } \\
\text { Integrated Approach }\end{array}$ & Gap \\
\hline $4 \times 4$ & 314.11 & 310.26 & $1.23 \%$ \\
$10 \times 6$ & 587.71 & 566.02 & $3.69 \%$ \\
$15 \times 8$ & 1803.44 & 1681.17 & $6.78 \%$ \\
$20 \times 10$ & 2668.54 & 2416.36 & $9.45 \%$ \\
\hline
\end{tabular}

${ }^{1}$ Initial depot assignments are generated randomly 


\section{Appendix I:}

Optimal solution for M5

\section{SOLVE SUMMARY}

$\begin{array}{llll}\text { MODEL } & \text { M5 } & \text { OBJECTIVE } & z \\ \text { TYPE } & \text { MINLP } & \text { DIRECTION } & \text { MINIMIZE } \\ \text { SOLVER } & \text { BARON } & \text { FROM LINE } & 74\end{array}$

\section{**** SOLVER STATUS \\ **** MODEL STATUS \\ **** OBJECTIVE VALUE}

Total time elapsed:
--- VAR $x_{10}$

--- VAR $x_{21}$

--- VAR $x_{42}$

--- VAR $x_{03}$

---- VAR $x_{34}$

---- VAR $y_{41}$

---- VAR $y_{32}$

---- VAR $y_{13}$

---- VAR $y_{24}$

--- VAR $u_{1}$

--- VAR $u_{2}$

--- $\quad$ VAR $u_{3}$

--- $\quad$ VAR $u_{4}$

--- VAR $z$
DIRECTION MINIMIZE

\author{
1 NORMAL COMPLETION \\ 1 OPTIMAL \\ 310.26
}

000:00:02, in seconds: 1.68

LOWER LEVEL UPPER MARGINAL

$\begin{array}{llll}. & 1.000 & 1.000 & 50.00 \\ \cdot & 1.000 & 1.000 & 58.42 \\ . & 1.000 & 1.000 & 89.44 \\ . & 1.000 & 1.000 & 53.98 \\ \cdot & 1.000 & 1.000 & 58.42 \\ . & 1.000 & 1.000 & 58.42 \\ . & 1.000 & 1.000 & 89.44 \\ . & 1.000 & 1.000 & 53.98 \\ . & 1.000 & 1.000 & 58.42 \\ 1.000 & 4.000 & 4.000 & \text { EPS } \\ 1.000 & 3.000 & 4.000 & \text { EPS } \\ 1.000 & 1.000 & 4.000 & \text { EPS } \\ 1.000 & 2.000 & 4.000 & \text { EPS } \\ - \text { INF } & 310.26 & + \text { INF } & .\end{array}$




\section{Appendix II:}

Optimal solution for M6

\section{SOLVE SUMMARY}

\section{MODEL M6 \\ TYPE MIP \\ SOLVER CPLEX \\ **** SOLVER STATUS \\ $* * * *$ MODEL STATUS \\ **** OBJECTIVE VALUE}

Total time elapsed:
OBJECTIVE $\quad z$

DIRECTION MINIMIZE

FROM LINE 273

1 NORMAL COMPLETION

1 OPTIMAL

310.26

000:00:00, in seconds: 0.16

LOWER LEVEL UPPER MARGINAL

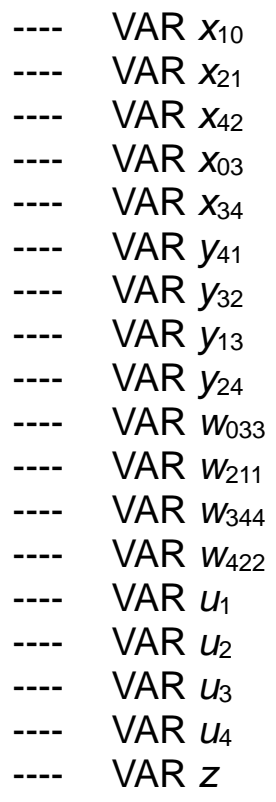

$\begin{array}{lll}1.000 & 1.000 \quad 50.000\end{array}$

$\begin{array}{lll}1.000 & 1.000 \quad \text { EPS }\end{array}$

$1.000 \quad 1.000 \quad$ EPS

$\begin{array}{ll}1.000 & 1.000 \quad \text { EPS }\end{array}$

$1.000 \quad 1.000 \quad$ EPS

$1.000 \quad 1.000 \quad$ EPS
1.000

$\begin{array}{lll}1.000 & 1.000 \quad \text { EPS }\end{array}$

$\begin{array}{lll}1.000 & 1.000 \quad 53.98\end{array}$

$\begin{array}{lll}1.000 & 1.000 \quad 58.42\end{array}$

$\begin{array}{lll}1.000 & 1.000 \quad 58.42\end{array}$

$\begin{array}{lll}1.000 & 1.000 \quad 89.44\end{array}$

$4.000 \quad 4.000 \quad$ EPS

$3.000 \quad 4.000 \quad$ EPS

$1.000 \quad 4.000 \quad$ EPS

$2.000 \quad 4.000 \quad$ EPS $\begin{array}{lll}1.000 & 1.000 \quad \text { EPS }\end{array}$

$1.000 \quad 1.000$ EPS

$\begin{array}{llll}1.000 & 2.000 & 4.000 & \text { EPS }\end{array}$

- INF $\quad 310.26 \quad+$ INF 